\title{
Robert J. Lempert* \\ Embedding (some) benefit-cost concepts into decision support processes with deep uncertainty
}

\begin{abstract}
Benefit-cost analysis (BCA) aims to help people make better decisions. But BCA does not always serve this role as well as intended. In particular, BCA's aim of aggregating all attributes of concern to decision makers into a single, bestestimate metric can conflict with the differing world views and values that may be an inherent characteristic of many climate-related decisions. This paper argues that new approaches exist that can help reduce the tension between the benefits of providing useful, scientifically based information to decision makers and the costs of aggregating uncertainty and differing values into single best estimates. Enabled by new information technology, these approaches can summarize decision-relevant information in new ways. Viewed in this light, many limitations of BCA lie not in the approach itself, but with the way it is used. In particular, this paper will argue that the problem lies in a process that begins by first assigning agreed-upon values to all the relevant inputs and then using BCA to rank the desirability of alternative decision options. In contrast, BCA can be used as part of a process that begins by acknowledging a wide range of ethical and epistemological views, examines which combinations of views are most important in affecting the ranking among proposed decision options, and uses this information to identify and seek consensus on actions that are robust over a wide range of such views.
\end{abstract}

Keywords: deep uncertainty; multi-attribute decision making; resilient infrastructure; robust decision making; sea level rise.

\section{Introduction}

Benefit-cost analysis (BCA) aims to help people make better decisions. Under appropriate circumstances, individuals and organizations whose choices

*Corresponding author: Robert J. Lempert, Rand Corporation, Santa Monica, CA 90401, USA, e-mail: Lempert@rand.org 
conform to those suggested by BCA would allocate resources most efficiently and thus achieve more of their goals than those whose choices deviated from those recommended by BCA. But under some conditions, BCA does not serve this decision-improving role as well as intended. Sussman, Grambsch, Li and Weaver (2014) note in their introduction to this special issue that applying BCA to climate-related decisions can involve numerous challenges such as what factors to include in the accounting of benefits and costs, how to value intangibles for which no market values exist, how to balance among current and future costs and benefits, and how to treat uncertainty about the future. While users and practitioners of BCA often face such questions, climate change makes the questions particularly salient because this policy domain involves a strong global externality; raises profound issues of intergenerational equity; and involves potentially large changes to physical, biological, and human systems that are impossible to predict with any confidence.

To some extent, technical improvements to BCA methodologies can help address the challenges of applying the approach to climate-related decisions. For instance, Neumann and Strzepek (2014) reviews the state of the art in the economic estimation of sectoral impacts of climate change and describes steps for improving such estimates. Weyant (2014) similarly offers suggestions for improving the integrated assessment models used to estimate the social cost of carbon, an important input to BCA-based regulatory impacts analysis. But at best, such technical improvements, while certainly valuable, provide only a partial solution. Climate change presents what some literature calls a "wicked" problem, one that is not well bounded, is framed differently by various groups and individuals, involves large scientific to existential uncertainties, and tends not to be well understood until after the formulation of a solution (Jones et al., 2014; Rittel \& Webber, 1973). BCA offers a particular framing of the climate change challenge, which involves aggregating all its disparate effects into a single quantitative measure and then using this measure to rank alternative solutions. As Toman (2014) notes in his contribution to this special issue, other ethical frameworks exist for viewing the climate change challenge that cannot be subsumed into the BCA framing. As a consequence, those who value these other ethical frameworks may not find compelling many BCA-based rankings of alternative responses to climate change.

The concept of "deep uncertainty" represents one important component of wicked problems and a particular focus of this essay. In technical terms, deep uncertainty exists when the parties to a decision do not know, or do not agree on, the system model that relates their actions to their consequences or the prior probability distributions for key inputs to such models (Lempert, Popper, \& Bankes, 2003). As generally practiced, BCA uses the formalisms of probabilistic 
decision analysis (Morgan \& Henrion, 1990) to address uncertainty, assuming that it is well characterized; that is, it can be usefully described by a single joint probability distribution over future states of the world. These distributions can be derived either from quantitative analysis or through expert elicitation of subjective (Bayesian) judgments of experts. This treatment of uncertainty is consistent with the overall BCA goal of aggregating all available information into a single quantitative measure.

While useful for many types of decision challenges, treating uncertainty as well characterized when it is in fact deep can lead to poor choices (Lempert, Nakicenovic, Sarewitz, \& Schlesinger, 2004; Lempert \& Popper, 2005; Morgan, Kandilikar, Risbey, \& Dowlatabadi, 1999; Sarewitz \& Pielke, 2000; Weaver et al., 2013). An analysis that portrays deep uncertainty as well characterized may encourage decision makers toward overconfidence and prompt them to choose strategies that fail when the future unfolds differently than expected (see for instance, Taleb, 2007). Presenting uncertainty as well characterized may also make it more difficult for parties with differing expectations about the future to reach consensus on a decision. Basing policy recommendations on forecasts can encourage those who oppose the recommendation to attack the forecast, which under conditions of deep uncertainty may prove more difficult to justify than the policy recommendation itself. In addition, the process of seeking a forecast on which one can base a policy recommendation may exclude information that is not useful for predicting but can nonetheless help identify, compare, and evaluate potential solutions. ${ }^{1}$

Recent critiques of the integrated assessment models used to inform climaterelated decisions exemplify how deep uncertainty can exacerbate the broader framing challenges of wicked problems. Rosen and Guenther (2014) and Pindyck (2013) argue that the projections produced by current models are so inaccurate as to be useless for any type of BCA analysis. They thus suggest framing the climate change challenge as a precautionary problem. But as noted by Weyant (2014) in this special issue, rejecting the models entirely is surely an overreaction, since they contain a wealth of useful information. More broadly, good decisions regarding complicated challenges such as climate change require solid grounding in quantitative, scientifically informed analyses within a well-structured framework, such as that embodied in BCA. Structured analysis can help individuals overcome numerous shortcomings and biases in unaided human reasoning

1 This loss of information in forecasts represents an example of what March and Simon (1958). call "uncertainty absorption," in which inferences are drawn from a body of evidence and the inferences rather than the evidence itself are communicated, severely limiting the ability of the recipient to judge the correctness of the inference. 
(Kahneman, 2011). Similarly, at the organizational level, Mishan notes that democratic decision making is time consuming and often prone to error. He highlights the value gained from expert best estimates of optimal resource allocations, as provided by BCA analyses (Mishan, 1994, pp. 159-166).

Overall, applying BCA to climate-related decisions has both benefits and costs. How then to best employ this structured analytic framework in this complex realm of climate change, fraught with deep uncertainties and profound disagreement over values?

This paper argues that the best answer to this question involves re-framing the way it is often cast. Rather than reject BCA entirely or focus solely on improvements to its methods for quantification, this paper argues for reorganizing how the different components of BCA methodologies are used to inform decision processes. In particular, new methods and tools exist for decision support that can help reduce the tension between the benefits providing well-structured, scientifically based information to decision makers and the costs of aggregating deep uncertainties and differing values into single best estimates. Enabled by new information technology, these approaches can summarize decision-relevant information in new ways.

Viewed in this light, many limitations on applying BCA to climate-related decisions lie not in the approach itself, but the way it is used. Traditionally, BCA follows a process that begins by first assigning agreed-upon values to all the relevant inputs and then using a benefit-cost criterion to rank the desirability of alternative decision options. In contrast, the elements of BCA can be used as part of a process that begins by acknowledging a wide range of ethical and epistemological stances, examining which combinations of expectations and values are most important in affecting the ranking among proposed decision options, and using this information to identify and seek consensus on actions that are robust over a wide range of ways of viewing the world.

\section{Employing BCA methods and tools in conditions of deep uncertainty}

Traditional probabilistic decision and risk analysis (Jones et al., 2014; Morgan \& Henrion, 1990; Moss et al., 2014), and with them BCA, follow what can usefully be described as an "agree-on-assumptions" process (Kalra et al., 2014). The analysis begins by first seeking agreement on assumptions regarding current and future conditions, and then ranks decision options contingent on these assumptions. In recent years, there has emerged a set of decision analytic methods for 
decision making under conditions of deep uncertainty (Cox, 2012; Kwakkel, Walker, \& Marchau, 2010) that follow the reverse process. These "agree-on-decisions" approaches defer any agreement on assumptions until decision options have been analyzed under many alternative sets of expectations and values. ${ }^{2}$ In general, such approaches aim not to provide a single "best" solution to decision makers, but rather help people manage wicked problems by facilitating participatory processes and interactions among analysts and decision makers. This essay will focus on one such “agree-on-decision” approach, Robust Decision Making (RDM) (Hallegatte, Shah, Lempert, Brown, \& Gill, 2012; Lempert, Groves, Popper, \& Bankes, 2006; Lempert et al., 2003). The comments here, however, often prove generally true of the broader class of methods.

RDM rests on a simple concept. ${ }^{3}$ Rather than using computer models and data to describe a best-estimate future, RDM runs models over hundreds to thousands of different sets of assumptions to describe how plans perform in many plausible futures. The approach then uses statistics and visualizations on the resulting large database of model runs to help decision makers distinguish those future conditions where their plans will perform well from those in which they will perform poorly. This information can help decision makers identify, evaluate, and choose robust strategies - ones that perform well over a wide range of futures and that better manage surprise.

As with other "agree-on-decision" approaches, RDM avoids the challenges faced by traditional BCA by incorporating the concept of running the analysis "backwards." Rather than start with predictions, the approach begins with one or more plans under consideration (often a current or best-estimate plan) and uses multiple runs of the model to identify the futures most relevant to the plan's success. The concept of stress-testing policies against scenarios is clearly not new. But new capabilities in information technology help RDM facilitate such reasoning. Just as people routinely use search engines like Google to scan millions of websites to find information that interests them, so RDM uses computer search and visualization on databases of many model runs to help decision makers to address such questions as: What are the key characteristics that differentiate those futures in which a plan succeeds from those in which it fails? What steps can be taken so a plan may succeed over a wider range of futures?

To understand the relationship between BCA and RDM, it is useful to first situate them both in the larger concept of decision support, and then within that

2 The literature offers several names for these "agree-on-decisions" approaches, including "context-first” (Ranger et al., 2010), “decision-scaling” (Casey, Brown, \& Wilby, 2012), and "assess risk of policy” (Carter et al., 2007; Dessai \& Hulme, 2007; Lempert et al., 2004).

3 These two paragraphs draw on concepts and some phrases from Lempert et al. (2013c). 
context compare distinct attributes of the two approaches. Decision support, using a common definition from the National Research Council (2009), represents the "set of processes intended to create the conditions for the production and appropriate use of decision-relevant information.” As a key tenet, the decision support concept emphasizes that the way in which quantitative information is integrated into decision-making processes often proves as important as the information products themselves. With this in mind, it becomes useful to note that quantitative methods such as BCA and RDM have at least four distinct attributes:

1. The performance metric used to measure the consequences of alternative decisions,

2. The decision criteria used to rank alternative decisions according to those measures,

3. The characterization of uncertainty, and

4. The decision process into which the information generated by the approach is incorporated.

What is generally thought of as BCA combines specific choices for each of these attributes. It is also important to note that the characterization of uncertainty is intertwined with the decision process in which this information is employed.

First, BCA uses monetary values to measure the consequences of decisions. Much BCA methodology involves quantitative methods for consistently estimating costs and benefits and for converting disparate factors into a common monetized measure. For instance, applying BCA to some pollution control technology might require estimating the dollar cost of purchasing and operating the technology and then estimating the dollar value of the lives saved through its effective operation.

Second, BCA employs the criterion that benefits ought to exceed the costs. This criterion can be used as a threshold for categorizing projects as desirable or not. Alternatively, it can be used to rank alternative decisions so that the one whose benefits most exceed its costs is the most desirable.

Third, BCA generally assumes well-characterized uncertainty. In some cases, BCA uses single-valued best estimates of benefits and costs. More sophisticated applications characterize uncertainty in these values by representing costs and benefits with a probability density function. Such BCA exercises often emphasize uncertainty quantification (i.e., specifying appropriate probability distributions to represent the uncertainty), and uncertainty reduction (i.e., decreasing the variance in probabilistic estimates) as important precursors in the analysis.

Fourth, BCA generally envisions a process in which experts gather evidence, estimate costs, and then provide BCA rankings to decision makers for review and action. Many regulatory and government policy-making processes are structured this way. Experts assemble scientific and economic information, which they use 
to generate best estimates of benefits and costs. The experts then supply these estimates to decision makers, who make policy proposals, solicit public review, and then issue a decision.

Considering these attributes as distinct proves important, because the first two (expressing consequences in monetary values and valuing decisions whose benefits exceed their costs) can be usefully separated from the second two (assuming well-characterized uncertainty and providing authoritative best-estimate values to a decision process). In particular, RDM is quite catholic regarding measures, and its robustness criteria can easily incorporate BCA criteria as described below. Thus, considering these four attributes as distinct allows BCA's decision criterion and valuation methods to be incorporated into an RDM process, which may provide approaches more appropriate for informing decision under many conditions of deep uncertainty.

As shown in Figure 1, RDM's process begins with a decision-structuring exercise in which decision makers define the goals, values, uncertainties, and choices under consideration. A key step in this process identifies one or more policies that will be the focus of the initial iterations of the analysis. Analysts next use computer models to generate a large database of runs, where each such case represents the performance of a proposed policy in one plausible future. In a process called "scenario discovery" (Bryant \& Lempert, 2010b; Groves \& Lempert, 2007; Lempert, 2013) computer visualization and statistics on this database then help decision makers identify clusters representing scenarios that illuminate

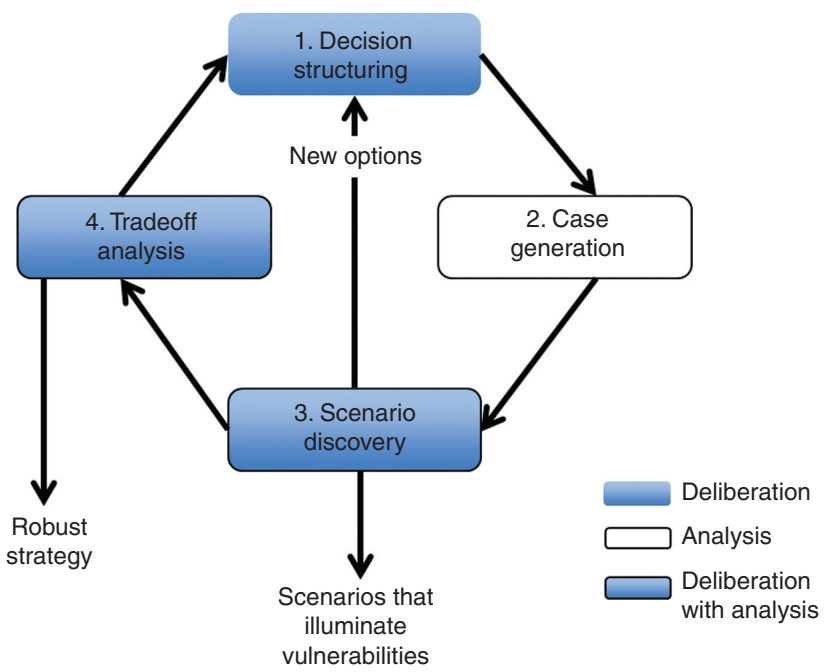

Figure 1 Iterative, participatory steps of an RDM analysis. 
vulnerabilities of the policies. These scenarios can then help decision makers identify potential new ways to address those vulnerabilities and evaluate through tradeoff analysis whether these choices are worth adopting. The process continues until decision makers settle on a robust strategy.

The literature has many definitions of robustness, but most can incorporate benefit-cost criteria. For instance, Lempert and Collins (2007) offer two definitions of robustness: 1) satisficing over a wide range of plausible futures and 2) trading some optimal performance for less sensitivity to broken assumptions. Both definitions can incorporate a benefit-cost criterion: the first by judging favorably decisions whose benefits exceed costs over a wide range of futures, and the second by judging favorably decisions whose expected benefit-cost ratio is close to optimum given a best estimate probability distribution over future states of the world, but remains satisfactory over a wide range of plausible probability density distributions. Note that robust strategies are often adaptive; that is, they are explicitly designed to evolve over time in response to new information (Rosenhead, 2001; Walker, Marchau, \& Swanson, 2010). The design of such strategies is often not obvious, but by providing detailed understanding of the vulnerabilities of proposed strategies, “agree-on-decisions" analytic methods often help decision makers identify and choose more successful adaptive strategies (Haasnoot et al., 2013; Lempert \& Groves, 2010; Lempert et al., 2003; Walker, Rahman, \& Cave, 2001).

RDM explicitly follows a "deliberation with analysis" process of decision support, in which parties to the decision deliberate on their objectives, options, and problem framing; analysts generate decision-relevant information using the system models; and the parties to the decision revisit their objectives, options, and problem framing influenced by this quantitative information (NRC, 2009). RDM adds to this general approach the concepts of running the analysis backward - that is, beginning with a proposed strategy - and testing plans against many different plausible futures. The overall process aims to facilitate deliberation among diverse stakeholders by embedding systematic quantitative reasoning about the consequences of and tradeoffs among alternative decision options within a framework that recognizes the legitimacy of different interests, values, and expectations about the future (Lempert, 2013; Lempert, Groves, \& Fischbach, 2013a; Parker, Srinivasan, Lempert, \& Berry, 2013).

RDM uses the same models and data as traditional BCA analyses, but in a fundamentally different way. The latter regard models as representations of reality that are sufficiently accurate to recommend the best response to an uncertain future. In contrast, RDM regards models as mapping assumptions to consequences (Bankes, 1993). Often RDM can significantly enhance the value of decision makers' existing models (designed for predict-then-act analysis) by 
running them numerous times to identify vulnerabilities and to find plans that are robust over many combinations of assumptions.

This RDM process can and does incorporate the qualitative measures and decision criterion on BCA, but in a context in which the costs and benefits are presumed to be deeply uncertain (Lempert, Schlesinger, \& Bankes, 1996; Toman, Griffin, \& Lempert, 2008). The next section will describe several examples in detail. The discussion below provides the basic outlines of the approach.

The RDM analysis would use a simulation model to evaluate the costs and benefits of one or more proposed policy or regulation contingent on the values for a large set of assumptions. These assumptions could involve differing expectations about future states of the world, differing interpretations of current observations or scientific theories, as well as differing opinions on how to value particular factors, such as the value of a life. The RDM analysis would then run this model thousands to millions of times to create a large database. Each entry in the database (a case) would report the monetized costs and benefits for one proposed policy for one particular set of values for each of the assumptions. (Each such set of assumptions is called a future.) BCA criteria would then be used to categorize each case as satisfactory (e.g., benefits exceed costs) or unsatisfactory (e.g., costs exceed benefits). Analysts could then use visualizations and statistics on this database to address questions such as:

- What policies meet the BCA criteria over a wide range of futures and values?

- What key combinations of assumptions are most important in distinguishing those futures where a particular policy meets the BCA criteria from those futures where it does not?

This information can then be used by analysts, or in deliberative decision processes with decision makers, to address other questions, such as: Does the balance of scientific evidence suggest that the combinations of assumptions that favor a policy are sufficiently likely so that decision makers should adopt the policy? Are there ways to modify a proposed policy, perhaps by allowing it to adjust over time, to significantly expand the set of assumptions over which it meets the BCA criteria?

\section{Example application for infrastructure investments at the Port of Los Angeles}

This RDM process has been employed in many applications. For instance, analytic facilitation work with the U.S. Bureau of Reclamation's recent Colorado 
Basin Supply and Demand Study (Groves, Fischbach, Bloom, Knopman, \& Keefe, 2013) and with the Louisiana Office of Coastal Projection and Restoration (Fischbach et al., 2012; Groves, Sharon, \& Knopman, 2012; IPCC 2014, see box 2.1), have helped large stakeholder groups with diverse values, interests, and expectations develop and come to consensus on robust plans. This paper, however, describes a much simpler application for the Port of Los Angeles that explicitly used a straightforward, science-rich application of BCA within an RDM framework (Lempert, Sriver, \& Keller, 2012; henceforth LSK).

The Port of Los Angeles (PoLA) is one of the largest container ship facilities in the world. Along with many jurisdictions worldwide, PoLA must consider how to include climate change - in particular sea level rise - into its infrastructure investment decisions. In particular, PoLA faces the question of how to address the potential for presumably low-probability but large-impact levels of extreme sea level rise in its investment plans. Such extreme events - e.g., increased storm frequency and/or a rapid increase in the rate of sea level rise - can affect infrastructure investments, but in ways difficult to address because of the deep uncertainty involved.

The LSK study thus used RDM to examine whether PoLA should consider hardening its container terminals against extreme sea level rise. ${ }^{4}$ Every few decades the port conducts a major upgrade of its terminals. During such an upgrade, the cost for hardening against an additional meter or so of sea level rise is relatively small. Hardening a terminal at other times would prove much more costly. Because these terminals are relatively high above the water, a decision to harden at the next upgrade can be thought of as purchasing relatively low-cost insurance against the potential impacts of poorly understood extreme events.

Should PoLA buy such insurance? To address this question, LSK conducted a BCA within an RDM framework. It proves useful to describe this analysis using the four attributes described above.

\subsection{Performance metric}

First, LSK uses a monetary performance metric to measure the consequences of the alternative decisions. LSK calculates these costs using a simple decision tree, as shown in Figure 2. Along the upper branch, PoLA chooses to harden a terminal at the next upgrade, which, by assumption, eliminates any subsequent impacts from sea level rise for the lifetime of the terminal. Thus the costs along

4 This section draws heavily from LSK. 


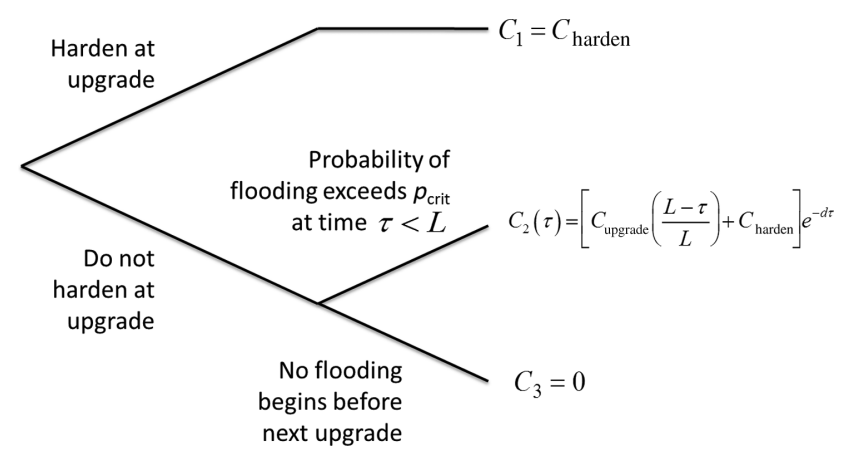

Figure 2 Simplified representation of PoLA's decision regarding whether to harden its terminal at its next upgrade, and the costs resulting from its choices from Lempert et al. (2012).

this branch are the monetary costs of hardening. Along the lower branch, PoLA chooses not to harden and the combination of future sea level rise and increased storm surge frequency proves insufficient to flood the terminal. For this branch there are no costs. Along the middle branch, PoLA decides not to harden and the combination of future sea level rise and storm surge frequency proves sufficient to flood the terminal. For this branch, the costs are the monetized damages due to flooding and the future costs of preventing those damages. For simplicity, LSK estimates these costs by assuming that there is some threshold probability of annual flooding beyond which PoLA will choose to replace its terminal with one that cannot be flooded. That is, LSK assumes that the primary cost of deciding not to harden at the next upgrade is the need for early replacement of an expensive asset. Overall, it is important to note that this analysis considers costs from the point of view of the financial perspective of the port, and not the broader social interest.

Figure 2 shows the resulting costs for each the three branches. The cost of the upper branch is the cost of hardening at the time of the upgrade, given by $C_{\text {harden }}$. The cost of the lower branch is zero. The cost of the middle branch is the present value of the lost lifetime of the terminal, given by $C_{\text {uprade }}\left(\frac{L-\tau}{L}\right) e^{-d \tau}$ where $\mathrm{L}$ is the terminal lifetime, $\tau$ the year is which PoLA choose to replace the terminal, and $d$ is the discount rate. This formulation of the benefit-cost calculation of course represents a significant simplification, but proves sufficiently rich for our purposes here. In particular, this problem formulation captures the fundamental tradeoff between buying inexpensive insurance today avoid potentially large future costs (note that $C_{\text {harden }}>>C_{\text {upgrade }}$ ) in the face of deep uncertainty regarding climate change and other trends. 


\subsection{Decision criterion}

As its decision criterion, LSK uses least expected cost. Accordingly, PoLA should choose to harden at the next upgrade if the cost of hardening is less than the expected cost of early terminal replacement forced by flooding in the future. That is, if

$$
C_{\text {harden }}<C_{\text {upgrade }} \int_{\tau=1}^{\tau=L} \rho\left(\tau, p_{\text {crit }}\right)\left(\frac{L-\tau}{L}\right) e^{-d \tau} d \tau
$$

where $\rho\left(\tau, p_{\text {crit }}\right)$ is the probability that PoLA will have to replace the terminal in year $\tau$ because the annual flooding probability has exceeded $p_{\text {crit }}$, the value PoLA managers in that year regard as a safe threshold. ${ }^{5}$

\subsection{Uncertainty characterization}

Many uncertainties affect this calculation of expected cost. The characterization of these uncertainties depends on the decision process employed. Traditional BCA assumes well-characterized uncertainty, and would begin by quantifying the uncertainty for each of the terms in Equation 1. In particular, analysts would use the best available science to generate probabilistic estimates of future sea level rise and of future changes in storm surge at the port in order to arrive at a best-estimate distribution for $\rho\left(\tau, p_{\text {crit }}\right)$. The available climate science is, however, insufficient to provide much confidence in such an estimate, especially in the tails of the distribution for the extreme levels of sea level rise that are most important to informing this particular decision.

The RDM process provides sufficient flexibility so that the uncertainty characterization can treat parameters in different ways, depending on the information available about them. LSK divides the parameters in Equation 1 into three categories, those: 1) whose values would be known at the time of the decision, 2) whose uncertainty is well characterized, and 3) whose uncertainty is deep. The first set of parameters has numeric values and the second has joint probability distributions. For the third set, LSK builds simple phenomenological models, scales over wide parameter ranges, identifies thresholds relevant to the BCA criteria, and then compares these thresholds to available scientific information. This process is described below. Here the focus is on the phenomenological models.

5 Note that Equation 1 could more generally be written as the integral over a joint probability distribution capturing uncertainties in $C_{\text {upgrade }}, L$, and $p_{\text {crit }}$ in addition to $\tau$. The RDM analysis described below will address uncertainty in all these parameters. 
Consistent with the risk management literature, this analysis considers three components of risk: hazard, exposure, and vulnerability. The parameters in Equation 1 related to future terminal management affect vulnerability, in particular the terminal lifetime $\mathrm{L}$ and the acceptable annual flooding likelihood $p_{\text {crit }}{ }^{*}$ Future climate conditions contribute to hazard, in particular the magnitude and timing of extreme sea level rise and any increase in storm surge frequency. The exposure is constant, because LSK consider a particular PoLA terminal.

LSK assumed that the cost of hardening, the cost of the upgrade, and the discount rate are all known at the time of the decision. A single, uncontroversial choice of discount rate was appropriate in this analysis because it compares the financial consequences of present and future capital investment decisions for a single decision-making entity.

LSK regards the two parameters associated with future terminal management the terminal lifetime and $p_{\text {crit }}$ - as deeply uncertain and considers a wide range of values for each.

To include the climate information in the analysis, LSK describes a simple model that scales over a wide range of climate futures in order to find the important thresholds for the BCA criteria, similarly to the way climate information is used in the decision-scaling approach (Brown, Werick, Leger, \& Fay, 2010; Brown \& Wilby, 2012). LSK then compares these thresholds, as described below, to the available science. LSK begins by considering two contributions to the hazard: annual mean sea level rise and the hourly anomaly in the sea level. LSK usefully approximates the annual mean sea level as:

$$
z_{t}=a+b t+c t^{2}+c^{\star} I\left(t-t^{\star}\right)
$$

where the term $a$ is the current sea level at PoLA, $b$ is a constant rate [mm/year], and $c$ is an acceleration term (mm/year ${ }^{2}$ ). To simplify the analysis, LSK assumes that these first three terms represent only the effects of relatively well-understood processes, such as thermal expansion of the oceans due to rising temperatures and the melting of small glaciers, that are well constrained by past observations. The fourth term represents currently poorly understood and poorly constrained processes, for example potentially abrupt changes in the dynamics of ice flow (cf. Alley, Clark, Huybrechts, \& Joughin, 2005), which LSK approximates with a step-function increase in the rate of sea-level rise $c^{\star}[\mathrm{mm} /$ year] that occurs after some time $t^{*}$. LSK regards the first three terms as well-characterized uncertainty, and fit a joint probability distribution for their values to past trends. LSK regards the two parameters governing the fourth term $-c^{\star}$ and $t^{*}-$ as deeply uncertain.

To estimate the hourly anomaly, LSK notes that PoLA has 80 years of hourly data on the sea level at the site of its terminals. These data are well approximated 
by a generalized extreme value (GEV) distribution. To consider potential changes in the frequency of large storm surge events, LSK considers a set of GEV distributions, created by varying the parameter governing the width of the current distribution over a wide range. ${ }^{6}$ LSK regards this change in the hourly anomaly as deeply uncertain.

\subsection{Process}

This RDM BCA is structured around asking two questions in the following order:

- Under what future conditions would a decision to harden at the next upgrade pass a benefit-cost test; that is, under what conditions is $C_{\text {harden }}<C_{\text {upgrade }}\left(\frac{L-\tau}{L}\right) e^{-d \tau}$ ?

- Do current science and other available information suggest that such conditions are sufficiently like to justify such an investment?

It proves useful to address these two questions sequentially because the first can be answered with confidence, while the second requires judgments that will intertwine expectations about future climate with values regarding what risks are most appropriate to take.

To identify the scenarios in which hardening at the next upgrade passes a benefit-cost test, LSK begins by generating a large number of cases (Step 2 in Figure 1). The study uses a 500-point Latin hypercube (LHC) sample over the five deeply uncertain parameters: three for future abrupt sea level rise and two for future terminal management. The LHC sample is similar to Monte Carlo, but provides a numerically more efficient sampling of the space defined by the deeply uncertain parameters. For each case in the sample, LSK calculates an expected cost by conducting a probabilistic sample over the parameters describing the well-characterized component of future sea level rise. This ensemble of 500 cases thus spans a wide range of futures characterized by both deep and well-characterized uncertainties.

The results, summarized in the left panel of Figure 3, show that in about two-thirds of the 500 cases a decision to harden at the next upgrade would fail a

\footnotetext{
6 Specifically, LSK varies the GEV scale parameter over the range $517 \mathrm{~mm} \leq \psi \leq 569 \mathrm{~mm}$, where the lower bound is the current scale and the upper bound is $10 \%$ larger. As the scale varies, the mean of the hourly anomaly around the annual mean must remain constant (our treatment of the anomalies demand that they do not shift the annual mean), so we write the location of each distribution in our set as $\mu=-176-(\psi-517 \mathrm{~mm})[\Gamma(1.305)-1]=-176+0.1033(\psi-517 \mathrm{~mm})$, where $\Gamma()$ is the gamma function (Hosking, 1990).
} 


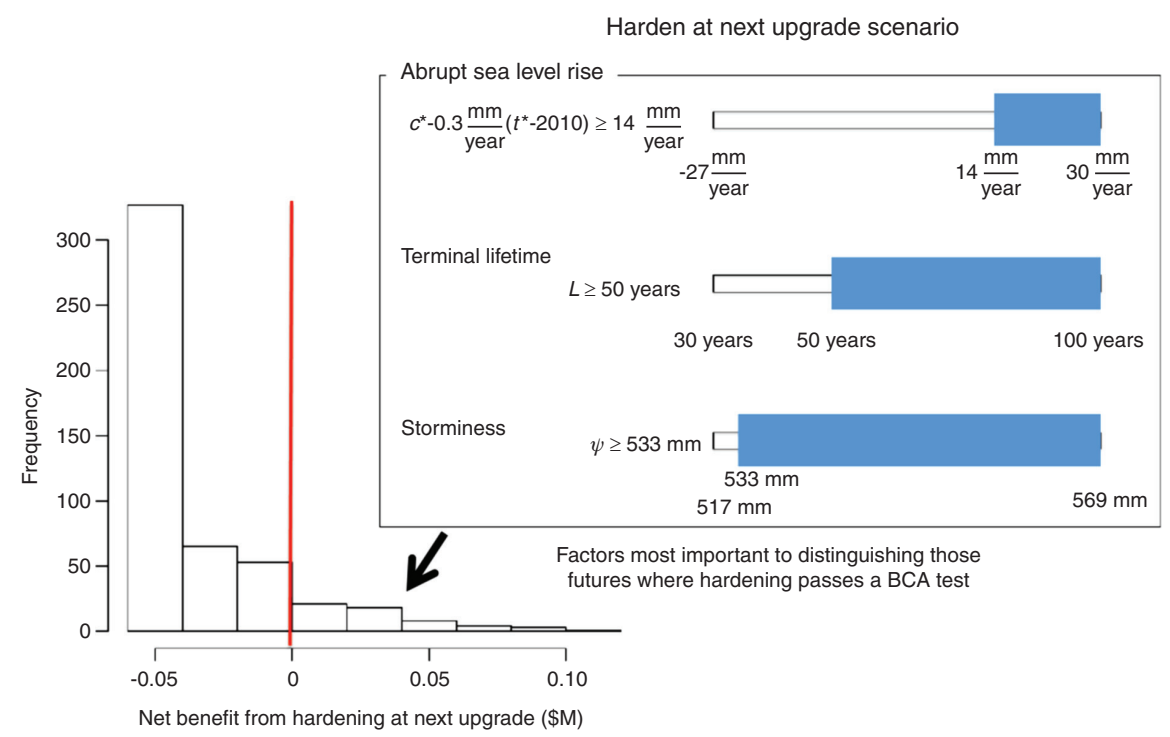

Figure 3 Histogram (left panel) showing net benefit for hardening for each of 500 alternative futures, and key factors (right panel) most important in distinguishing those futures where hardening passes a benefit-cost test from those futures in which it does not from Lempert et al. (2012).

benefit-cost test, while in the remaining one-third of cases hardening would have cost savings. In a small number of cases these savings would be quite large, up to 20 times the cost of the hardening.

The study next performs a statistical "scenario discovery" analysis on this database of model runs (Bryant \& Lempert, 2010; Dalal, Han, Lempert, Jaycocks, \& Hackbarth, 2013; Lempert et al., 2006) to answer the question: What conditions best characterize those futures where hardening at the next upgrade passes a benefit-cost test (Step 3 in Figure 1)? Scenario discovery applies a cluster analysis to the database, seeking to identify those combinations of uncertain input parameters that most concisely distinguish those futures in which the BCA is positive from those where it is negative. ${ }^{7}$ The results, shown in the right panel of Figure 3, suggest that hardening at the next upgrade makes sense in cases with near-term

7 The algorithms seek to maximize three measures of merit for the scenarios: coverage, which is the fraction of all vulnerable cases contained within the scenario; (2) density, which is the fraction of cases within the scenario that are vulnerable; and (3) interpretability: the ease with which the scenario can be communicated to and understood by policymakers. Interpretability is typically measured heuristically as the number of restrictions used to define the scenario. Improving any one of these three measures often negatively impacts one or both of the others. 
and rapid sea level rise, a long terminal lifetime, and a significant increase in local storminess. The specific values parameter part for these thresholds are shown on the figure. The value of the critical threshold proves less important.

LSK labels this cluster of cases the Harden at the Next Upgrade scenario. The study also calculates a probability threshold for this scenario; that is, the likelihood that a risk-neutral decision maker would have to ascribe to it such that the BCA decision criteria for hardening in Equation 1 is satisfied. Using a uniform distribution over the two respective sets of cases yields a probability threshold of $>7 \%$. That is, if the Harden at the Next Upgrade scenario is more likely than $7 \%$, then hardening may make sense.

Identifying this Harden at the Next Upgrade scenario and its probability threshold proves valuable because they represent quantitative statements that can be made with high confidence in this otherwise deeply uncertain situation. The analysis makes clear that if the conditions shown in Figure 3 hold, then the decision to harden at the next upgrade passes a benefit-cost test. The analysis also suggests that if a risk-neutral decision maker who estimated these conditions to be more likely than $7 \%$ then they should consider such hardening.

LSK now inquires the extent to which the available scientific evidence suggests that the Harden at the Next Upgrade scenario is sufficient likely to justify a decision to harden at the next upgrade (step 4 in Figure 1). The study begins by noting that definition of the scenario and its probability threshold imply hardening passes a benefit-cost test if the following conditions hold:

$$
\operatorname{Pr}\left\{c^{\star} \geq 14 \frac{\mathrm{mm}}{\text { year }}+0.3 \frac{\mathrm{mm}}{\text { year }}\left(t^{\star}-2010\right)\right\} \operatorname{Pr}\{\psi>533 \mathrm{~mm}\} \operatorname{Pr}\{L>50 \text { years }\}>7 \%
$$

Sufficient climate science is available to help inform imprecise probabilistic judgments about the likelihood of the parameters describing abrupt sea level rise. First note that the first scenario condition in Equation 3 implies a sea level rise contribution from poorly understood processes of about 1.4 meter (m) in 2100. When combined with the roughly $0.5 \mathrm{~m}$ contribution from wellunderstood processes, the Harden at Next Upgrade scenario implies roughly two meters of sea level rise by century's end. Such a level is within, but at the high end of, some current sea level rise projections. To quantify this statement, LSK takes two expert assessments of the range of plausible sea level rise, fits probability distributions to these ranges, and then maps these distributions onto a joint probability distribution for the parameters $c^{\star}$ and $t^{\star}$.

For the two expert estimates, LSK draws on Pfeffer, Harper and O’Neel (2008) and the California Sea Level Rise Interim Guidance document CO-CAT (2010), and then uses a rejection sampling approach to generate a joint distribution for $c^{\star}$ 
and $t^{\star}$ consistent with each expert assessment. These two joint distributions then imply a range of probability estimates for the extreme sea level rise condition in Figure 3. The range is relatively narrow, and suggests that the probability that the extreme sea level rise condition for the Harden at the Next Upgrade scenario will be satisfied lies between 14 and 16\%.

Less information is available to estimate probability ranges for the other two scenario conditions in Equation 3. Figure 4 thus summarizes the decision-relevant constraints on these two conditions. The probability ranges on the first scenario conditions suggests that in order to satisfy Equation 3, the joint probability of the other two conditions should lie in the figure's shaded region; that is, the product of the two probabilities ought to exceed roughly $67 \%$.

First, note that the scenario condition on the lifetime is longer than those previously experienced by PoLA. While only sparse scientific evidence exists regarding the condition for the hourly anomaly, $\psi>533 \mathrm{~mm}$, none of it currently supports a high likelihood estimate for this scenario condition. Some studies (Menendez and Woodworth, 2010; Woodworth and Blackman, 2004) suggest that the future hourly anomaly may remain unchanged from that currently observed (i.e.: $\psi \approx 517 \mathrm{~mm}$ ). In contrast, other studies (Bromirski, Flick, \& Cayan, 2003; Méndez, Menéndez, Luceño, \& Losada, 2007) find that the observed variability of sea levels has been increasing at several locations. Cayan et al. (2008) analyze model projections and place bounds on future increases in storminess near San Francisco, corresponding in LSK's analysis to a range of $517 \mathrm{~mm} \leq \psi \leq 533 \mathrm{~mm}$. While future research may suggest these bounds are too

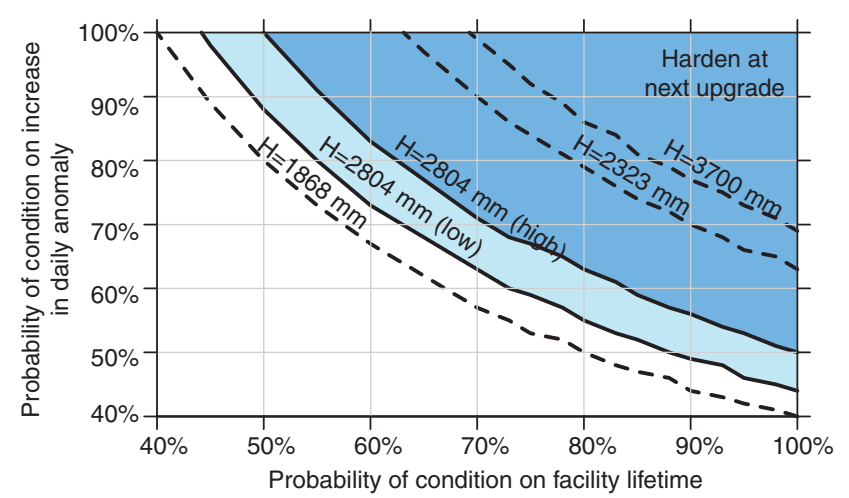

Figure 4 Probabilities of a long terminal lifetime ( $L>50$ years) and significant increase in the daily anomaly $(\psi>533 \mathrm{~mm})$ required for decision to harden terminal bottoms $(\mathrm{H}=2804 \mathrm{~mm})$ (at next upgrade to pass a cost-benefit test). Dark and light shaded regions show probabilities required using high and low estimates, respectively, of likelihood of condition on $c^{\star}$ and $t^{\star}$. Dashed lines show results for other facilities discussed in Lempert et al. (2012). 
narrow, ${ }^{8}$ current evidence does not support judgments that the likelihood of the future storminess scenario condition might lie in the region for hardening at the next upgrade shown in Figure 4.

Thus, PoLA might reasonably choose not to harden at the next upgrade of this facility, even at a cost of $1 \%$ of the cost of the upgrade. LSK repeated this analysis for three other PoLA assets. For one, the Alameda and Harry Bridges Crossing, hardening at the next upgrade might pass a benefit-cost test.

\section{Imprecise probabilities, threshold responses, and precaution}

Given the importance of probabilistic information to traditional BCA and the importance of critical thresholds and potentially irreversible impacts to many climate-related decisions, it is useful to review how the previous example dealt with these issues.

To represent deep uncertainty, RDM uses sets of alternative probability distributions over future states of the world (Morgan et al., 2009). That is, RDM considers imprecise probabilities (Walley, 1991). Consistent with the concept of backwards analysis, RDM analyses often report probability thresholds, representing the likelihood decision makers would have to ascribe to particular scenarios in order to change their choice of strategy. Figure 4 shows an example of such probability thresholds in the context of a decision that involves low-probability, high-consequence extreme events.

This approach to deeply uncertain probabilistic information offers at least three advantages (Lempert, 2013; Lempert et al., 2006). First, it offers a value of information ranking of the most important uncertainties for which probabilistic information would prove decision-relevant. This ranking can usefully focus efforts aimed at uncertainty quantification. Second, differing judgments about probabilities to the end of the analysis can help parties to the decision explore the implications of uncomfortable or contentious findings before committing themselves to the necessity of accepting their implications. This concept derives from scenario planning, which presents multiple views of the future without privileging among them, in order to expand the range of futures considered by decision makers and to facilitate communication and collaboration among individuals

8 Note that the values at the high end of LSK's experimental design range produce a storm surge of roughly 1.6 meters at the return rates of relevance to this analysis. In contrast, Hurricanes Ike and Katarina in the US Gulf Coast produced a maximum surge of up to 7 or 8 meters. 
with differing expectations and values (Schoemaker, 1993). This feature played an important role in successfully engaging port staff with the PoLA analysis, by allowing them to consider the vulnerability of their facilities to extreme sea level rise before having to make any judgments about whether they should take such vulnerabilities seriously. Third, considering a wide range of plausible probability distributions can help identify strategies whose good performance is insensitive to potentially erroneous probability judgments (Hall et al., 2012; Lempert \& Collins, 2007; McInerney, Lempert, \& Keller, 2012). Identifying such strategies may be particularly important in the face of potentially abrupt and irreversible changes in the climate system.

The precautionary principle offers another approach to addressing deeply uncertain probabilistic information. The principle suggests that an activity be avoided unless clear evidence exists that it will not prove harmful. In contrast, RDM addresses precaution in two distinct ways. First, the scenario discovery step in Figure 1 identifies the key conditions that one might wish to guard against, thus clarifying the conditions that might justify precaution. Second, the tradeoff analysis step in Figure 1 can use the definition of robustness that trades some optimal performance for less sensitivity to broken assumptions (Lempert \& Collins, 2007). This criterion interpolates between precaution (expressed as minimizing the maximum regret) and expected value, thus allowing decision makers to ask how much precaution they wish to adopt.

\section{Multi-attribute decision making}

The PoLA example demonstrates an application of BCA in an "agree-on-decision" framework that focuses on the treatment of deep uncertainty regarding the likely consequences of alternative decisions. This example considers a situation in which all the consequences, both present and future, of alternative policy choices are easily quantified into a common monetized metric. It is useful to briefly review other examples where this is not the case.

Multi-attribute decision theory (Keeney \& Raiffa, 1993), or multi-criteria decision analysis (MCDA), provides a general framework for comparing alternative policy choices based on their outcomes. This decision theory acknowledges that people may care about different potential consequences of their actions and may not find it useful to combine them all into a single quantitative metric. In general to compare alternative actions according to multiple objectives, one can: 1) quantify all the objectives using a single metric, 2) employ separate metrics for each type of consequence and apply a weighting to sum them in an aggregate index, or 
3) retain multiple metrics and evaluate policy options based on tradeoffs among these multiple objectives. BCA follows the first approach. Multi-attribute decision theory emphasizes the second two.

Some RDM analyses have employed the second approach, that of applying weightings to aggregate separate metrics into a single index. For instance, Lempert et al. (2003) compared alternative sustainable development policies using an index, inspired by the human development index (UNDP, 1990), that included individual metrics for per capita income, longevity, and environmental quality. To reflect alternative worldviews, the analysis considered four different weightings over each metric, differing in the weight placed on environmental quality (either zero weight or equal to the weight placed on income and longevity) and weight placed on the developed countries that were one main audience for the policy analysis (either population weighted over OECD countries only, or population weighted globally). The analysis separately identified robust strategies for each of the four different weightings. One weighting the one that included both global population averages and environmental quality - was most stressing in the sense that, of the adaptive decision strategies considered, the one that proved most robust using the global index with environmental quality was also robust for the other three indices. This most stressing index was thus the focus of the analyses. Note that the BCA analyses, as part of an effort to monetize costs or benefits, often make value judgments that may be contested by parties to the affected decisions. This general approach of seeking robust strategies over alternative weightings might thus provide another way to use BCA within an RDM framework in contexts where alternative choices about quantitative methodologies reflect real differences in ethical values.

Many RDM analyses compare policy options based on the third approach above, illuminating tradeoffs among multiple objectives. The "dashboard" approach to BCA advocated by Toman (2014) is one example of this approach to multi-criteria decision analysis. The RDM-based Colorado Basin Supply and Demand Study (Groves et al., 2013) considered 26 different measures including factors such as reliability of water deliveries, environmental projection, recreation opportunities, and cost, each evaluated at different geographic locations - as part of a multi-year process of deliberation with analysis with representatives of the federal government, seven Western states, and other users of the Colorado River system. The analysis presented the decision makers with tradeoff curves showing how alternative strategies balanced among these objectives over a wide range of plausible future scenarios. The high correlation among many of the objectives simplified the task of generating low-dimensional tradeoff curves that proved useful for the decision makers. 
Tools are increasingly becoming available to facilitate such deliberative processes in the more general case in which multiple objectives are not well correlated and it proves difficult to identify strategies robust across them. For instance, Kasprzyk, Nataraj, Reed and Lempert (2013) recently demonstrated an approach for identifying "Pareto satisficing" water management strategies using evolutionary algorithms and a simple measure of robustness. In multi-attribute decision theory, a set of Pareto-optimal strategies are those one whose performance according to any one objective cannot be improved without degrading performance according to any other objective, contingent on some best-estimate assumptions about the future. Pareto satisficing generalizes on this concept by seeking a set of strategies whose performance stays reasonably close to the Pareto optimal surfaces for each of many alternative future states of the world. Kasprzyk et al. (2013) operationalized this concept by using evolutionary algorithms to calculate a Pareto-optimal set of water management strategies, with a focus on the use of water markets, for a best-estimate probability distribution over future states of the world while considering multiple objectives including cost, cost variability, various measures of reliability, and complexity of the underlying water markets. The analysis estimated the robustness of this set of strategies to deep uncertainty by evaluating how much their performance, according to each objective, varied with alternative assumptions about the likelihood of important factors such as future extreme drought. The analysis found that the performance of some classes of strategies varied little over the full range of plausible futures, compared with that for strategies with more complex market designs. In particular, the analysis suggests that simple market designs prove more robust than more complex market designs. Overall, this example suggests how BCA can be used as one criterion within a multi-attribute analysis.

\section{Deliberation and delegation}

This paper argues that BCA concepts, used within an "agree-on-decision" process, can in some circumstances help people to make better decisions than they would using BCA in a more traditional form. This claim raises the question of what constitutes a "better decision." In some cases, we might regard a good decision as one that leads to a good outcome. But seemingly good choices can turn out badly, while seemingly unreasonable ones can turn out well. In principle, a good decision might correspond to some optimal choice derived from best scientific estimates of future uncertainty. But as documented in this special issue, this criterion often proves hard to implement for many types of climate-related decisions. 
In light of such considerations, Working Group II of the IPCC Fifth Assessment Report (2014, FAQ 2-1) provides a more process-oriented description of good decisions. The report notes that no universal criterion exists, but good decisions tend to emerge from processes in which people are:

- Explicit about their goals

- Consider a range of alternative options

- Consider tradeoffs

- Use best available science to understand the potential consequences of their actions

- Contemplate the decision from a wide range of views and vantages

- Follow agreed-upon rules and norms that enhance the legitimacy of the process and its outcomes

In the limit in which uncertainties are well characterized and goals aligned, these criteria suggest that the standard BCA process - which aggregates different measures into a single monetary value, summarizes uncertainties with a best-estimate probability distribution, ranks the benefit-cost ratios of alternative options, and then provides this information to decision makers - may lead to good decisions. However, in situations characterized by deep uncertainties and differing values, a backwards analysis embedded in a process of deliberation with analysis may prove more successful. Such backwards analyses can have costs. RDM in particular is often more demanding of computational resources than are traditional analyses, and often requires more complete systems models that relate actions to consequences (Lempert \& Collins, 2007; Lempert et al., 2013b). Thus, when uncertainties are well characterized, RDM may prove unnecessarily costly. But when uncertainties are deep, using BCA concepts with such a process may avoid overconfidence, may allow multiple entry points into the analysis, and may help widen both the range of uncertainties and the range of policy responses considered.

These claims are based on inferences from the psychological and organizational behavior literatures (see for instance Bryant \& Lempert, 2010; Lempert \& Popper, 2005; Lempert et al., 2003) and on experience gathered from many engagements using RDM in decision-support applications. However, formal testing and evaluation proves particularly useful toward determining the extent to which expectations about the effectiveness of decision-support methods such as RDM are borne out in practice. A handful of studies have evaluated components of the RDM process. Budescu, Lempert, Broomell \& Keller (2013) compared two decision aids that portray imprecise probabilities in different ways: one aid ranks decision options using expected values contingent on a best-estimate probability density function (consistent with traditional BCA) and the other aid 
graphically displays the expected value of the alternative decision options over the entire range of plausible probabilities (consistent with RDM). The experiment found that the type of aid systematically influenced the way subjects approached the problem, and that subjects had a preference for the latter aid. Parker et al. (2013) used experiments with individuals and expert interviews to evaluate understanding of and utility gained from the results of scenario discovery analyses. The study found that participants generally understand the scenario discovery displays. When these analytically derived scenarios were compared with related scenarios derived from a more traditional qualitative process, some experts found the former more difficult to understand. But those comfortable with the RDM process found its scenarios more solid, comprehensive, and concrete. While such studies yield valuable insights, more work in this vein would clearly be useful in suggesting the contexts in which alternative decision support methods can prove most useful and how they can be made more effective.

The last of the IPCC's criteria for an effective decision process suggests an additional challenge for using BCA within an "agree-on-decision” process: that existing rules and norms for some decision processes may favor "agreeon-assumptions” analyses. Many previous “agree-on-decision” applications have focused on deliberative processes, such as the planning efforts carried out by water and flood risk management agencies, in which the participants have considerable discretionary authority to weigh risks and to suggest appropriate policy responses. In such cases, the analysis aims to help these groups reach consensus and exercise their discretionary judgments more wisely. In contrast, public agencies often use BCA in a context in which experts exercise authority delegated to them by an elected authority and, as such, are trying to limit the scope of their discretionary judgment. In such cases, existing rules and norms may favor BCA's framework for providing best-estimate judgments of existing science, authoritative aggregations of differing values, and single rankings of alternative policy choices. Analytic processes that highlight the existence of multiple, legitimate views might not fit as easily within such contexts.

This mismatch between the benefits of an "agree-on-decisions" analytic process and the existing norms and rules of delegated decision processes might be reduced in several ways. Most straightforwardly, if "agree-on-decisions" methods allow an agency to identify a robust strategy - that is, one that meets its goals over a wide range of ways of aggregating values and of best-estimate judgments then the agency can report this result and demonstrate its insensitivity to the uncertainties. For instance, adaptive management strategies (Lempert \& Collins, 2007; Lempert et al., 1996), those designed to evolve over time in response to new 
information, may often prove more robust than static strategies. Such adaptive strategies can prove difficult to implement, but "agree-on-decision" methods may reduce some of these barriers (Lee, 1993). In other cases, in which no strategy is robust over the full range of expectations and values, "agree-on-decisions" analyses may provide transparency regarding the most important sets of assumptions. For instance, agencies basing a decision on a social cost of carbon estimate could report the sets of future conditions under which that decision would have turned out differently, or the regrets of choosing a higher social cost of carbon compared with the regrets of choosing a lower value. Good planning requires decision makers to consider the conditions in which their plan might not meet its goals. By using BCA within an "agree-on-decisions" approach, decision makers could make these judgments clear.

Overall, BCA provides a powerful set of tools for improving decisions, although in some cases the approach does not serve this role as well as intended. In particular, BCA's aim of aggregating all attributes of concern to decision makers into a single, best-estimate metric can conflict with the differing world views and values that may be inherent characteristics of many climate-related decisions. To help resolve such difficulties, one can usefully note that traditional BCA has a least four distinct attributes: (1) the cost and benefit performance metric used to compare the consequences of alternative decisions, (2) the decision criterion that ranks alternative decisions by the extent to which benefits exceed costs, (3) the characterization of the uncertainty that affects these rankings, and (4) a process that first seeks agreement on the assumptions that determine the estimates of costs and benefits before ranking alternative decisions according to these estimates. New information technology now makes it possible, however, to effectively employ BCA's metrics and decision criterion in alternative decision processes that may be better suited to many climate-related decisions. In particular, BCA's core concepts can be used within an "agreeon-decisions" analytic process that begins by acknowledging a wide range of ethical and epistemological views, examines which combinations of views are most important in affecting the ranking among proposed decision options, and uses this information to identify and seek consensus on actions that are robust over a wide range of such views.

Acknowledgments: The author thanks Klaus Keller and Ryan Sriver for their help in summarizing in Section 3 the work we did together. The author also thanks two anonymous reviewers, Anne Grambsch, and the other authors in this special issue, for their very helpful comments. This paper was supported in part by the Network for Sustainable Climate Risk Management (SCRiM) under NSF cooperative agreement GEO-1240507. 


\section{References}

Alley, R. B., Clark, P. U., Huybrechts, P., \& Joughin, I. (2005). Ice-sheet and sea-level changes. Science, 310(5747), 456-460.

Bankes, S. C. (1993). Exploratory modeling for policy analysis. Operations Research, 41(3), 435-449.

Bromirski, P. D., Flick, R. E., \& Cayan, D. R. (2003). Storminess variability along the California coast: 1858-2000. Journal of Climate, 16(6), 982-993.

Brown, C. (2010). The end of reliability. Journal of Water Resources Planning and Management, 136(2), 143-145.

Brown, C., \& Wilby, R. (2012). An alternate approach to assessing climate risks. Eos, Transations American Geophysical Union, 93(41), 401.

Brown, C., Werick, W., Leger, W., \& Fay, D. (2011). A decision analytic approach to managing climate risks - Application to the Upper Great Lakes. Journal of the American Water Resources Association 47(3), 524-534.

Bryant, B. P., \& Lempert, R. J. (2010). Thinking inside the box: A participatory, computer-assisted approach to scenario discovery. Technological Forecasting and Social Change, 77, 34-49.

Budescu, D. V., Lempert, R., Broomell, S., \& Keller, K. (2013). Aided and unaided decisions with imprecise probabilities. European Journal of Operational Research, 2(1-2), 31-62.

Carter, T. R., Jones, R. N., Lu, S. B. X., Conde, C., Mearns, L. O., O’Neill, B. C., ... Zurek M. B. (2007). New Assessment Methods and the Characterisation of Future Conditions. Climate Change 2007: Impacts, Adaptation and Vulnerability. Contribution of Working Group II to the Fourth Assessment Report of the Intergovernmental Panel on Climate Change. M. L. Parry, O. F. Canziani, J. P. Palutikof, P. J. v. d. Linden and C. E. Hanson (Eds.). Cambridge, UK: Cambridge University Press, 1, 33-171.

Cayan, D. R., Bromirski, P. D., Hayhoe, K., Tyree, M., Dettinger, M. D., \& Flick, R. E. (2008). Climate change projections of sea level extremes along the California coast. Climatic Change, 87(1), 57-73.

CO-CAT. (2010). "State of California Sea-Level Rise Interim Report." Retrieved December 11, 2014, from http://www.opc.ca.gov/webmaster/ftp/project_pages/Climate/SLR_ Guidance_Document.pdf.

Collins, M. (2007). Ensembles and probabilities: A new era in the prediction of climate change. Philosophical Transactions of the Royal Society A, 365, 1957-1970.

Cox, J., Louis Anthony (Tony). (2012). Confronting deep uncertainties in risk assessment. Risk Analysis, 32(10), 1607-1629.

Dalal, S., Han, B., Lempert, R. J., Jaycocks, A., \& Hackbarth, A. (2013). Improving scenario discovery using orthogonol rotations. Environmental Modeling and Software, 48, 1-16.

Dessai, S., \& Hulme, M. (2007). Assessing the robustness of adaptation decisions to climate change uncertainties: A case study on water resources management in the east of England. Global Environmental Change, 17(1), 59-72.

Fischbach, J. R., Johnson, D. R., Ortiz, D. S., Bryant, B. P., Hoover, M., \& Ostwald, J. (2012). Coastal Louisiana risk assessment model. Santa Monica, CA: RAND Gulf States Policy Institute.

Groves, D. G., \& Lempert, R. J. (2007). A New Analytic Method for Finding Policy-Relevant Scenarios. Global Environmental Change, 17, 73-85.

Groves, D. G., Sharon, C., \& Knopman, D. (2012). Planning tool to support Louisiana's decisionmaking on coastal protection and restoration. Santa Monica, CA: RAND Gulf States Policy Institute. 
Groves, D. G., Fischbach, J. R., Bloom, E., Knopman, D., \& Keefe, R. (2013). Adapting to a changing Colorado River: Making future water deliveries more reliable through robust management strategies. Santa Monica, CA: RAND Corporation.

Haasnoot, M., Kwakkel, J. H., Walker, W. E., \& ter Maat, J. (2013). Dynamic adaptive policy pathways: A new method for crafting robust decisions for a deeply uncertain world. Global Environmental Change, 23(2), 485-498.

Hall, J. M., Lempert, R., Keller, K., Hackbarth, A., Mijere, C., \& McInerney, D. (2012). Robust climate policies under uncertainty: A comparison of Info-Gap and RDM methods. Risk Analysis, 32(10), 1657-1672.

Hallegatte, S., Shah, A., Lempert, R. J., Brown, C., \& Gill, S. (2012). Investment decision making under deep uncertainty: Application to climate change. Washington, DC: World Bank.

Hosking, J. R. M. (1990). L-moments - Analysis and estimation of distribution using linear- combinations of order statistics. Journal of the Royal Statistical Society Series B-Methodological 52(1), 105-124.

IPCC. (2007). Climate change 2007: Impacts, adaptation and vulnerability. Working Group II contribution to the Fourth Assessment Report of the Intergovernmental Panel on Climate Change. T. R. Carter, R. N. Jones, S. B. X. Lu, C. Conde, L. O. Mearns, \& B. C. O’Neill (Eds.). Cambridge, UK: Cambridge University Press.

IPCC. (2014). Climate change 2014: Impacts, adaptation, and vulnerability. Contribution of Working Group II to the Fifth Assessment Report of the Intergovernmental Panel on Climate Change. C. B. Field, V. R. Barros, D. J. Dokken, K. J. Mach, M. D. Mastrandrea, T. E. Bilir, ... L. L. White (Eds.). Cambridge, UK and New York, NY: Cambridge University Press.

Jones, R. N., Patwardhan, A., Cohen, S., Dessai, S., Lammel, A., Lempert, R. J., ... van Storch, H. (2014). Chapter 2. Foundations for decision making. In Intergovernmental Panel on Climate Change (IPCC) (Ed.), Climate Change 2014: Impacts, Adaptation, and Vulnerability. Cambridge, UK and New York, NY: Cambridge University Press.

Kahneman, D. (2011). Thinking, fast and slow. New York: Farrar, Straus and Giroux.

Kalra, N., Hallegatte, S., Lempert, R. J., Brown, C., Fozzard, A., Gill, S., \& Shah A. (2014). Agreeing on robust decisions: A new process for decision making under deep uncertainty. Washington, DC: Policy Research Working paper, World Bank.

Kasprzyk, J. R., Nataraj, S., Reed, P. M., \& Lempert, R. J. (2013). Many-objective robust decision making for complex environmental systems undergoing change. Environmental Modeling and Software, 42, 55-71.

Keeney, R. L., \& Raiffa, H. (1993). Decisions with multiple objectives. Cambridge, UK: Cambridge University Press.

Kwakkel, J., Walker, W., \& Marchau, V. (2010). Classifying and communicating uncertainties in model-based policy analysis. International Journal of Technology Management, 10(4/2010), 1468-4322.

Lee, K. (1993). Compass and gyroscope: Integrating science and politics for the environment. Washington, DC: Island Press.

Lempert, R. J. (2013). Scenarios that illuminate vulnerabilities and robust responses. Climatic Change, 117, 627-646.

Lempert, R. J., \& Popper, S. W. (2005). High-performance government in an uncertain world. In R. Klitgaard \& P. Light (Eds.), High Performance Government: Structure, Leadership, and Incentives. Santa Monica, CA: RAND Corporation.

Lempert, R. J., \& Collins, M. (2007). Managing the risk of uncertain threshold responses: Comparison of robust, optimum, and precautionary approaches. Risk Analysis, 27(4), 1009-1026. 
Lempert, R. J., \& Groves, D. G. (2010). Identifying and evaluating robust adaptive policy responses to climate change for water management agencies in the American West. Technological Forecasting and Social Change, 77, 960-974.

Lempert, R. J., Schlesinger, M. E., \& Bankes, S. C. (1996). When we don't know the costs or the benefits: Adaptive strategies for abating climate change. Climatic Change, 33(2), 235-274.

Lempert, R. J., Popper, S. W., \& Bankes, S. C. (2003). Shaping the next one hundred years: New methods for quantitative, long-term policy analysis. Santa Monica, CA: RAND Corporation.

Lempert, R. J., Nakicenovic, N., Sarewitz, D., \& Schlesinger, M. (2004). Characterizing climatechange uncertainties for decision-makers - An editorial essay. Climatic Change, 65(1-2), 1-9.

Lempert, R. J., Groves, D. G., Popper, S. W., \& Bankes, S. C. (2006). A general, analytic method for generating Robust strategies and narrative scenarios. Management Science, 52(4), 514-528.

Lempert, R. J., Sriver, R. L., \& Keller, K. (2012). Characterizing uncertain sea level rise projections to support investment decisions. Sacramento, CA: California Energy Commission.

Lempert, R. J., Groves, D. G., \& Fischbach, J. R. (2013a). Is it ethical to use a single probability density function? Santa Monica, CA: RAND Corporation.

Lempert, R. J., Kalra, N., Peyraud, S., Mao, Z., Tan, S. B., Cira D., \& Lotsch A. (2013b). Ensuring robust flood risk management in Ho Chi Minh City: A robust decision making demonstration. Washington, DC: World Bank.

Lempert, R. J., Popper, S. W., Groves, D. G., Kalra, N., Fischbach, J. R., Bankes, S. C., ... McInerney D. J. (2013C). Making good decisions without predictions: Robust decision making for planning under deep uncertainty. Santa Monica, CA: RAND Corporation.

March, J., \& Simon H. (1958). Organizations. Oxford, England: John Wiley.

McInerney, D., Lempert, R.J., \& Keller, K. (2012). What are robust strategies in the face of uncertain climate threshold responses? Robust Climate Strategies. Climate Change, 112(3-4), 547-568.

Méndez, F. J., Menéndez, M., Luceño, A., \& Losada, I. J. (2007). Analyzing monthly extreme sea levels with a time-dependent GEV model. Journal of Atmospheric and Oceanic Technology, 24(5), 894-911. doi:10.1175/JTECH2009.1.

Menendez, M., \& Woodworth P. L. (2010). Changes in extreme high water levels based on a quasi-global tide-gauge data set. Journal of Geophysical Research-Oceans 115.

Mishan, E. J. (1994). Cost-benefit analysis. London, UK: Routledge.

Morgan, M. G., \& Henrion, M. (1990). Uncertainty: A guide to dealing with uncertainty in quantitative risk and policy analysis. Cambridge, UK: Cambridge University Press.

Morgan, M. G., Kandilikar, M., Risbey, J., \& Dowlatabadi, H. (1999). Why conventional tools for policy analysis are often inadequate for problems of global change. Climatic Change, 41, 271-281.

Morgan, M. G., Dowlatabadi, H., Henrion, M., Keith, D., Lempert, R. J., McBride, S., ... Wilbanks, T. (2009). Best practice approaches for characterizing, communicating, and incorporating scientific uncertainty in decisionmaking. Synthesis and Assessment Product 5.2.

Moss, R., Scarlett, P. L., Kenney, M. A., Kunreuther, H., Lempert, R., Manning, J., \& Williams, B.K. (2014). Decision support: Connecting science, risk perception, and decisions. Washington DC: US Global Change Research Program.

National Research Council. (2009). Informing decisions in a changing climate. Washington, DC: The National Academies Press. 
Neumann, J. E., \& Strzepek K. (2014). State of the literature on the economic impacts of climate change in the United States. Journal of Benefit-Cost Analysis, 5(3), 411-443.

Parker, A. M., Srinivasan, S., Lempert, R. J., \& Berry, S. (2013). Evaluating simulation-derived scenarios for effective decision support. Technological Forecasting \& Social Change, in press.

Pfeffer, W. T., Harper, J. T., \& O’Neel, S. (2008). Kinematic constraints on glacier contributions to 21st-century sea-level rise. Science, 321(5894), 1340-1343.

Pindyck, R. (2013). Climate change policy: What do the models tell us? Journal of Economic Literature, 51(3), 860-872.

Ranger, N., Millner, A., Dietz, S., Fankhauser, S., Lopez, A., \& Ruta, G. (2010). Adaptation in the UK: A decision making process. London: Granthan/CCEP Policy Brief.

Rittel, H., \& Webber, M. (1973). Dilemmas in a general theory of planning. Policy Sciences, 4, 155-169.

Rosen, R. A., \& Guenther, E. (2014). The economics of mitigating climate change: What can we know? Technological Forecasting \& Social Change. In press.

Rosenhead, J. (2001). Robustness analysis: Keeping your options open. In Johnathan Rosenhead \& J. Mingers (Eds.), Rational Analysis for a Problematic World Revisited. Chichester, UK: John WIley and Sons.

Sarewitz, D., \& R. A. Pielke, J. (2000). Science, prediction: Decisionmaking, and the future of nature. Washington, DC: Island Press.

Schoemaker, P. J. H. (1993). Multiple scenario development: Its conceptual and behavioral foundation. [Abstract]. Strategic Management Journal, 14(3), 193-213.

Sussman, F., Grambsch, A., Li J., \& Weaver C. P. (2014). Introduction to a special issue entitled Perspectives on Implementing Benefit-Cost Analysis in Climate Assessment. Journal of Benefit-Cost Analysis, 5(3), 333-346.

Taleb, N. N. (2007). The black swan: The impact of the highly improbable. New York: Random House.

Toman, M. (2014). The need for multiple types of information to inform climate change assessment. Journal of Benefit-Cost Analysis, 5(3), 469-485.

Toman, M. A., Griffin, J., \& Lempert, R. J. (2008). Impacts on U.S. energy expenditures and greenhouse-gas emissions of increasing renewable-energy use: Technical report (No. 9780833044976 (pbk. alk. paper)). Santa Monica, CA: RAND Corporation.

United Nations Development Programme (UNDP). (1990). Human development report. Oxford, UK: Oxford University Press.

Walker, W., Marchau, V., \& Swanson, D. (2010). Addressing deep uncertainty using adaptive policies. Technology Forecasting and Social Change, 77, 917-923.

Walker, W. E., Rahman, S. A., \& Cave, J. (2001). Adaptive policies, policy analysis, and policymaking. European Journal of Operational Research, 128, 282-289.

Walley, P. (1991). Statistical reasoning with imprecise probabilities. London: Chapman and Hall.

Weaver, C. P., Lempert, R. J., Brown, C., Hall, J. A., Revell, D., \& Sarewitz, D. (2013). Improving the contribution of climate model information to decision making: the value and demands of robust decision frameworks. WIREs Climate Change, 4,39-60.

Weyant, J. (2014). Integrated assessment of climate change: state of the literature. Journal of Benefit-Cost Analysis, 5(3), 377-409.

Woodworth, P. L., \& Blackman D. L. (2004). Evidence for systematic changes in extreme high waters since the mid-1970s. J Climate 17(6), 1190-1197. 